\title{
On Analytic Solutions of (1+3)D Relativistic Ideal Hydrodynamic Equations
}

\author{
Shu Lin ${ }^{\mathrm{a}, 1}$, Jinfeng $\operatorname{Liao}^{\mathrm{b}, 2}$ \\ ${ }^{a}$ Department of Physics \& Astronomy, SUNY Stony Brook, NY11794, USA. \\ ${ }^{b}$ Nuclear Science Division, Lawrence Berkeley National Laboratory, \\ MS70R0319, 1 Cyclotron Road, Berkeley, CA 94720, USA.
}

\begin{abstract}
In this paper, we find various analytic $(1+3) \mathrm{D}$ solutions to relativistic ideal hydrodynamic equations based on embedding of known low-dimensional scaling solutions. We first study a class of flows with 2D Hubble Embedding, for which a single ordinary differential equation for the remaining velocity field can be derived. Using this equation, all solutions with transverse 2D Hubble embedding and power law ansatz for the remaining longitudinal velocity field will be found. Going beyond the power law ansatz, we further find a few solutions with transverse $2 \mathrm{D}$ Hubble embedding and nontrivial longitudinal velocity field. Finally we investigate general scaling flows with each component of the velocity fields scaling independently, for which we also find all possible solutions.
\end{abstract}

Key words: Hydrodynamics, Relativistic Heavy Ion Collisions PACS: 47.75.+f, 25.75.-q, 24.10.Nz

\footnotetext{
${ }^{1}$ slin@grad.physics.sunysb.edu

2jliao@lbl.gov
} 


\section{Introduction}

Relativistic hydrodynamics [1, 2] is widely used in physics. Its particular application to high energy collision dynamics can be dated back to Laudau and Khalatnikov [3] in 1950's. For a brief history and an introduction to this aspect, see e.g. [4]. The relativistic ideal hydrodynamics (RIHD) has played a significant role in the past decade for the development of theory and phenomenology of the quark-gluon plasma (QGP) [5] - a deconfined high temperature phase of QCD matter. The QGP can be experimentally created and studied by the Relativistic Heavy Ion Collisions (RHIC). The space-time evolution at RHIC is characterized by fast longitudinal expansion (longitudinal flow) and strong transverse expansion (radial and elliptic flow). In particular, the large elliptic flow $v_{2}$ in non-central collisions [6] can be well described by RIHD [7] at low-tointermediate transverse momenta for almost all particle species and for various centralities, beam energies and colliding nuclei, implying very low viscosity of the underlying matter, see reviews in e.g. [9, 10]. It is now widely believed that the QGP in $1-2 T_{c}$ region (as covered by current RHIC energy) is a "nearly perfect fluid" 11 12] (see however caveats as discussed in e.g. [13 14]). The success of RIHD description for RHIC space-time evolution has become the basis for the paradigm shift that the QGP in $1-2 T_{c}$ region is actually a stronglycoupled liquid [15, 16]. Such strong coupling and good fluidity close to $T_{c}$ may have its origin in the magnetic component of QCD plasma [17, 18] which is ultimately connected with the mechanism of QCD deconfinement transition.

The success of RIHD has also inspired a lot of interest in the formal study of RIHD. Analytic solutions from RIHD have been very useful for RHIC phenomenology, for example the well-known Hwa-Bjorken boost invariant flow $[19,20]$. There have been many efforts and progresses in finding analytic solutions, with an emphasis on solutions with potential importance for describing RHIC collisions. Study aiming at $(1+1)$ D solutions that give an alternative description of the longitudinal expansion and a more realistic (nonboost-invariant) multiplicity distribution over rapidity can be found in e.g. 21, 22, 23, 24]. General Solutions of the $(1+1)$ D RIHD with arbitrary initial conditions in the forward light-cone for specific equation of states are also found in [21]. Beyond (1+1)D, ellipsoidally expanding flows have been studied in [25] [26], and generalization of the spherically symmetric Hubble flows to accelerating, non-Hubble flows in arbitrary $(1+\mathrm{d}) \mathrm{D}$ has been done in 21]. There have also been study of adding radial flow to a longitudinal Bjorken profile in e.g. 27], and more recently attempts to deal with elliptic flow analytically [28]. A new method for solving relativistic ideal hydrodynamics in $(1+3) \mathrm{D}$ is developed in 29], where by explicitly embedding in the longitudinal and transverse radial flows, the authors reduce the RIHD equations to a single equation for the transverse velocity field only and find analytically all possible solutions with their transverse velocity fields having power dependence on proper time and transverse radius.

In this paper, we aim to find analytic solutions to RIHD in $(1+3) \mathrm{D}$, by taking the approach of Liao and Koch in 29]. Basically one uses lower-dimensional scal- 
ing solutions (Hubble flow in 1D and 2D) to build solutions in higher-dimension. We will first study a class of flows with 2D Hubble Embedding, for which a single ordinary differential equation for the remaining velocity field will be derived in Section-3. Using this equation, all solutions with transverse 2D Hubble embedding and power law ansatz for the remaining velocity field will be found in Section-4. More interesting solutions with transverse 2D Hubble embedding and nontrivial longitudinal flow beyond power law ansatz will be investigated and found in Section-5. In Section-6 we will study general scaling flows with each component of the velocity fields scaling independently, for which we also find all possible solutions. Finally we summarize and discuss the results in Section-7.

\section{Generalities}

We start with the relativistic hydrodynamics equations:

$$
T^{m n} ; n=0
$$

For ideal hydrodynamics, the energy-momentum tensor is given by

$$
T^{m n}=(\epsilon+p) u^{m} u^{n}-p g^{m n}=w u^{m} u^{n}-p g^{m n}
$$

with $\epsilon, p$ and $w=\epsilon+p$ being the energy density, pressure and the enthalpy density defined in the flowing matter's local rest frame (L.R.F) which by definition are Lorentz scalars. The flow field $u^{m}(x)$ is constrained by $u^{m} \cdot u_{m}=1$. In the usual $(t, \vec{x})$ coordinates one can express $u^{m}(x)$ as $\gamma(1, \vec{v})$ with $\gamma=1 / \sqrt{1-\vec{v}^{2}}$ and $\vec{v}=d \vec{x} / d t$.

To close the set of equations for five independent fields $\epsilon, p, \vec{v}$, we also need Equation of State (E.o.S)

$$
p=\nu(\epsilon+p)=\nu w
$$

The above means $\epsilon=\frac{1-\nu}{\nu} p$ implying a speed of sound $c_{s}=\sqrt{\frac{\partial p}{\partial \epsilon}}=\sqrt{\frac{\nu}{1-\nu}}$, and, in order to assure $c_{s} \leq 1$, we require $0<\nu \leq 1 / 2$.

\subsection{Curved coordinates for 2D Hubble Embedding}

For a 2D Hubble Embedding study, we will use a coordinate system of $\left(\tau_{\perp}, \eta_{\perp}, \phi_{\perp}, z\right)$ : i.e. the transverse proper time, the spatial transverse rapidity, the transverse angle, and the longitudinal $z$. They are related to the usual $(t, x, y, z)$ in the following way (with $\left.r_{\perp} \equiv \sqrt{x^{2}+y^{2}}\right)$ :

$$
\begin{aligned}
& \tau_{\perp}=\sqrt{t^{2}-r_{\perp}^{2}}, \eta_{\perp}=\frac{1}{2} \ln \frac{t+r_{\perp}}{t-r_{\perp}}, \\
& \phi_{\perp}=\frac{1}{2 i} \ln \frac{x+y \cdot i}{x-y \cdot i}, z=z
\end{aligned}
$$

and inversely

$$
\begin{aligned}
& t=\tau_{\perp} \cosh \eta_{\perp}, z=z, \\
& x=\tau_{\perp} \sinh \eta_{\perp} \cos \phi_{\perp}, y=\tau_{\perp} \sinh \eta_{\perp} \sin \phi_{\perp}
\end{aligned}
$$


The velocity field $u^{m}$ in these coordinates is related to $u^{\mu}=\gamma\left(1, v_{x}, v_{y}, v_{z}\right)$ in flat coordinates $(t, x, y, z)$ via

$$
\begin{aligned}
& u^{\tau_{\perp}}=\gamma\left[\cosh \eta_{\perp}-\left(v_{x} \cos \phi_{\perp}+v_{y} \sin \phi_{\perp}\right) \sinh \eta_{\perp}\right] \\
& u^{\eta_{\perp}}=\frac{\gamma}{\tau_{\perp}}\left[\left(v_{x} \cos \phi_{\perp}+v_{y} \sin \phi_{\perp}\right) \cosh \eta_{\perp}-\sinh \eta_{\perp}\right] \\
& u^{\phi_{\perp}}=\frac{\gamma}{\tau_{\perp} \sinh \eta_{\perp}}\left(v_{y} \cos \phi_{\perp}-v_{x} \sin \phi_{\perp}\right) \\
& u^{z}=\gamma v_{z}
\end{aligned}
$$

The metric tensor associated with the $\left(\tau_{\perp}, \eta_{\perp}, \phi_{\perp}, z\right)$ coordinates is

$$
\begin{aligned}
& g_{m n}=\operatorname{Diag}\left(1,-\tau_{\perp}^{2},-\tau_{\perp}^{2} \sinh ^{2} \eta_{\perp},-1\right) \\
& g^{m n}=\operatorname{Diag}\left(1,-\frac{1}{\tau_{\perp}^{2}},-\frac{1}{\tau_{\perp}^{2} \sinh ^{2} \eta_{\perp}},-1\right)
\end{aligned}
$$

With the metric above, it can be explicitly checked that $u^{m} u_{m}=g_{m n} u^{m} u^{n}=1$.

For the covariant derivatives in the hydrodynamic equations Eq.(11), we will need the Affine connections $\Gamma_{m n}^{j}=g^{j k} \Gamma_{k m n}=g^{j k} \frac{1}{2}\left(g_{k m, n}+g_{k n, m}-g_{m n, k}\right)$. In our case the non-vanishing connections are:

$$
\begin{aligned}
& \Gamma_{\eta_{\perp} \eta_{\perp}}^{\tau_{\perp}}=\tau_{\perp}, \Gamma_{\eta_{\perp} \tau_{\perp}}^{\eta_{\perp}}=\Gamma_{\tau_{\perp} \eta_{\perp}}^{\eta_{\perp}}=\frac{1}{\tau_{\perp}} \\
& \Gamma_{\phi_{\perp} \phi_{\perp}}^{\tau_{\perp}}=\tau_{\perp} \sinh ^{2} \eta_{\perp}, \Gamma_{\tau_{\perp} \phi_{\perp}}^{\phi_{\perp}}=\Gamma_{\phi_{\perp} \tau_{\perp}}^{\phi_{\perp}}=\frac{1}{\tau_{\perp}} \\
& \Gamma_{\phi_{\perp} \phi_{\perp}}^{\eta_{\perp}}=-\sinh \eta_{\perp} \cosh \eta_{\perp}, \Gamma_{\eta_{\perp} \phi_{\perp}}^{\phi_{\perp}}=\Gamma_{\phi_{\perp} \eta_{\perp}}^{\phi_{\perp}}=\frac{\cosh \eta_{\perp}}{\sinh \eta_{\perp}}
\end{aligned}
$$

\section{2D Hubble Embedding and Derived Velocity Equation}

Now to find solutions in $(1+3) \mathrm{D}$ space-time, we first do an explicit 2D Hubble embedding, which involves the following ansatz for the transverse part of the velocity field:

$$
v_{x}=\frac{x}{t}=\tanh \eta_{\perp} \cos \phi_{\perp}, v_{y}=\frac{y}{t}=\tanh \eta_{\perp} \sin \phi_{\perp}
$$

which in the $\left(\tau_{\perp}, \eta_{\perp}, \phi_{\perp}, z\right)$ coordinates translate nicely into:

$$
\begin{array}{r}
u^{\tau_{\perp}}=\frac{\gamma}{\cosh \eta_{\perp}}, u^{\eta_{\perp}}=0 \\
u^{\phi_{\perp}}=0,, u^{z}=\gamma v_{z}
\end{array}
$$

The above can be further re-written as:

$$
u^{m}=\bar{\gamma}\left(1,0,0, \bar{v}_{z}\right)
$$

by introducing $\bar{v}_{z}=v_{z} \cosh \eta_{\perp}, \bar{\gamma}=\sqrt{1-\bar{v}_{z}^{2}}$. 
Now inserting the velocity field Eq.(14) together with the metric Eq.(10) and the E.o.S Eq.(3) (which substitutes the $p$ by $\nu w$ ) into the energy-momentum tensor in Eq.(2), we can explicitly obtain all the non-vanishing components to be:

$$
\begin{aligned}
& T^{\tau_{\perp} \tau_{\perp}}=\left[-1+\frac{\bar{\gamma}^{2}}{\nu}\right] p \\
& T^{\tau_{\perp} z}=\frac{\bar{\gamma}^{2} \bar{v}_{z}}{\nu} p \\
& T^{\eta \eta}=\frac{p}{\tau_{\perp}^{2}} \\
& T^{\phi \phi}=\frac{p}{\tau_{\perp}^{2} \sinh ^{2} \eta_{\perp}} \\
& T^{z z}=\left[1+\frac{\bar{\gamma}^{2} \bar{v}_{z}^{2}}{\nu}\right] p
\end{aligned}
$$

From the above, we can explicitly write down the hydrodynamic equations below:

$$
\begin{aligned}
& {\left[-\nu \tau_{\perp}+\bar{\gamma}^{2} \tau_{\perp}\right] p,_{\tau_{\perp}}+\bar{\gamma}^{2} \tau_{\perp} \bar{v}_{z} p, z} \\
& \quad+\left[2 \bar{\gamma} \tau_{\perp}\left(\bar{\gamma}, \bar{\tau}_{\perp}+\bar{v}_{z} \bar{\gamma}, z\right)+\bar{\gamma}^{2}\left(2+\tau_{\perp} \bar{v}_{z, z}\right)\right] p=0 \\
& p,_{\eta_{\perp}}=0 \\
& p,_{\phi_{\perp}}=0 \\
& \bar{\gamma}^{2} \bar{v}_{z} \tau_{\perp} p_{\tau_{\perp}}+\left[\bar{\gamma}^{2} \bar{v}_{z}^{2} \tau_{\perp}+\nu \tau_{\perp}\right] p,_{z} \\
& \quad+\left[2 \bar{\gamma} \bar{v}_{z}\left(\bar{\gamma},_{\perp}+\bar{\gamma}, \bar{v}_{z}\right) \tau_{\perp}+\bar{\gamma}^{2}\left(\tau_{\perp} \bar{v}_{z, \tau_{\perp}}+2 \bar{v}_{z}\left(1+\tau_{\perp} \bar{v}_{z, z}\right)\right)\right] p=0
\end{aligned}
$$

The two equations about $\eta_{\perp}$ and $\phi_{\perp}$ are trivially solved by setting all fields independent of these two variables, i.e. $p\left(x^{m}\right)=p\left(\tau_{\perp}, z\right)$ and $\bar{v}_{z}\left(x^{m}\right)=\bar{v}_{z}\left(\tau_{\perp}, z\right)$. Then we can convert the remaining two equations into the following:

$$
\begin{aligned}
& (\ln p)_{, \tau_{\perp}} \equiv \mathcal{F}\left[\tau_{\perp}, z\right] \\
& =\frac{-\tau_{\perp} \bar{v}_{z}\left(\bar{v}_{z, \tau_{\perp}}+\bar{v}_{z} \bar{v}_{z, z}\right)+\nu\left[2\left(1-\bar{v}_{z}^{2}\right)+2 \tau_{\perp} \bar{v}_{z} \bar{v}_{z, \tau_{\perp}}+\tau_{\perp}\left(1+\bar{v}_{z}^{2}\right) \bar{v}_{z, z}\right]}{(-1+\nu) \nu \tau_{\perp}\left(1-\bar{v}_{z}^{2}\right)^{2}} \\
& (\ln p)_{, z} \equiv \mathcal{G}\left[\tau_{\perp}, z\right] \\
& =\frac{\tau_{\perp}\left(\bar{v}_{z, \tau_{\perp}}+\bar{v}_{z} \bar{v}_{z, z}\right)+\nu\left[-2 \bar{v}_{z}\left(1-\bar{v}_{z}^{2}\right)-\tau_{\perp}\left(1+\bar{v}_{z}^{2}\right) \bar{v}_{z, \tau_{\perp}}-2 \tau_{\perp} \bar{v}_{z} \bar{v}_{z, z}\right]}{(-1+\nu) \nu \tau_{\perp}\left(1-\bar{v}_{z}^{2}\right)^{2}}
\end{aligned}
$$

The commutativity of partial derivative $(\ln p)_{, \tau_{\perp}, z}=(\ln p)_{, z, \tau_{\perp}}$ leads to the following constraint which is a derived equation only involving the velocity field: 


$$
\begin{aligned}
& \tau_{\perp}^{2}\left[\bar{v}_{z, \tau_{\perp}, \tau_{\perp}}+2 \bar{v}_{z, \tau_{\perp}} \bar{v}_{z, z}-2 \bar{v}_{z}^{3}\left(\bar{v}_{z, \tau_{\perp}, z}-\bar{v}_{z, z}^{2}\right)+2 \bar{v}_{z}\left(2 \bar{v}_{z, \tau_{\perp}}^{2}+\bar{v}_{z, \tau_{\perp}, z}+\bar{v}_{z, z}^{2}\right)\right. \\
& \left.-\bar{v}_{z}^{4} \bar{v}_{z, z, z}+\bar{v}_{z}^{2}\left(-\bar{v}_{z, \tau_{\perp}, \tau_{\perp}}+6 \bar{v}_{z, \tau_{\perp}} \bar{v}_{z, z}+\bar{v}_{z, z, z}\right)\right] \\
& +\nu\left[2 \bar{v}_{z}^{5}-12 \tau_{\perp}^{2} \bar{v}_{z}^{2} \bar{v}_{z, \tau_{\perp}} \bar{v}_{z, z}-2 \bar{v}_{z}^{3}\left(2-2 \tau_{\perp} \bar{v}_{z, z}+\tau_{\perp}^{2}\left(\bar{v}_{z, \tau_{\perp}}^{2}-2 \bar{v}_{z, \tau_{\perp}, z}+\bar{v}_{z, z}^{2}\right)\right)\right. \\
& -2 \bar{v}_{z}\left(-1+2 \tau_{\perp} \bar{v}_{z, z}+\tau_{\perp}^{2}\left(3 \bar{v}_{z, \tau_{\perp}}^{2}+2 \bar{v}_{z, \tau_{\perp}, z}+3 \bar{v}_{z, z}^{2}\right)\right)+\tau_{\perp} \bar{v}_{z}^{4}\left(2 \bar{v}_{z, \tau_{\perp}}+\right. \\
& \left.\left.\tau_{\perp}\left(\bar{v}_{z, \tau_{\perp}, \tau_{\perp}}+\bar{v}_{z, z, z}\right)\right)-\tau_{\perp}\left(\bar{v}_{z, \tau_{\perp}}\left(2+4 \tau_{\perp} \bar{v}_{z, z}\right)+\tau_{\perp}\left(\bar{v}_{z, \tau_{\perp}, \tau_{\perp}}+\bar{v}_{z, z, z}\right)\right)\right] \\
& =0
\end{aligned}
$$

Once the above derived velocity equation has been solved, the matter field can be obtained as:

$$
p=p_{0} \cdot \operatorname{Exp}\left[\int_{\tau_{\perp 0}}^{\tau_{\perp}} d \tau_{\perp}{ }^{\prime} \mathcal{F}\left[\tau_{\perp}{ }^{\prime}, z\right]+\int_{z_{0}}^{z} d z^{\prime} \mathcal{G}\left[\tau_{\perp}, z^{\prime}\right]\right]
$$

with $p_{0}$ being the value at arbitrary reference point $\tau_{\perp 0}, z_{0}$.

\section{Analytic Solutions with Transverse 2D Hubble Embedding and Longitudinal Power Law Ansatz}

As an application of the derived velocity equation (26) in the previous section, we now find all solutions with power law dependence on the variables $\tau_{\perp}$ and $z$. Within such ansatz of the velocity field

$$
\bar{v}_{z}=A \tau_{\perp}^{B} z^{C}
$$

Eq.(26) can be expressed explicitly as:

$$
\begin{aligned}
& \left(A C \nu-A C^{2} \nu\right) \tau_{\perp}^{2+B} z^{-2+C} \\
& +\left(-A B+A B^{2}+2 A \nu-A B \nu-A B^{2} \nu\right) \tau_{\perp}^{B} z^{C} \\
& +\left(A^{3} B+3 A^{3} B^{2}-4 A^{3} \nu-6 A^{3} B^{2} \nu\right) \tau_{\perp}^{3 B} z^{3 C} \\
& +\left(2 A^{5} \nu+A^{5} B \nu-A^{5} B^{2} \nu\right) \tau_{\perp}^{5 B} z^{5 C} \\
& +\left(4 A^{2} B C-4 A^{2} C \nu-8 A^{2} B C \nu\right) \tau_{\perp}^{1+2 B} z^{-1+2 C} \\
& +\left(-A^{3} C+3 A^{3} C^{2}-6 A^{3} C^{2} \nu\right) \tau_{\perp}^{2+3 B} z^{-2+3 C} \\
& +\left(4 A^{4} B C+4 A^{4} C \nu-8 A^{4} B C \nu\right) \tau_{\perp}^{1+4 B} z^{-1+4 C} \\
& +\left(A^{5} C+A^{5} C^{2}-A^{5} C \nu-A^{5} C^{2} \nu\right) \tau_{\perp}^{2+5 B} z^{-2+5 C}=0
\end{aligned}
$$

In the above equation, terms with various powers of $\tau_{\perp}, z$ (and only power terms) appear: to make all of them, either mutually cancel (among terms with exactly the same $\tau_{\perp}, \rho$ powers) or vanish by respective coefficients, to eventually zero is quite nontrivial. A thorough sorting of the sequences of $\tau_{\perp}, \rho$ powers can exhaust all possibilities to satisfy this algebraic equation. After enumerating all 
the possibilities, we find only four sets of solutions:

(i) $A=0$, with $x^{2}+y^{2}<t^{2}$;

(ii) $A=1, B=-1, C=1$, with $x^{2}+y^{2}+z^{2}<t^{2}$;

(iii) $A=1, B=1, C=-1$, with $x^{2}+y^{2}<t^{2}$ and $x^{2}+y^{2}+z^{2}>t^{2}$;

(iv) $A=-3, B=-1, C=1$, with $\nu=\frac{1}{2}$ and $x^{2}+y^{2}+9 z^{2}<t^{2}$.

The solution (i) is just the 2D Hubble expansion without z-motion, the solution (ii) is actually 3D Hubble expansion, while the remaining two are more nontrivial. The applicable kinematic domain for each solution is given above in terms of $(t, x, y, z)$ coordinates. The solution (iii) composed of two causally disconnected pieces: $z>\sqrt{t^{2}-x^{2}-y^{2}}$ and $z<-\sqrt{t^{2}-x^{2}-y^{2}}$. It can be considered as another type of explosion 3 , with the longitudinal flow being "anti-Hubble". The solution (iv) corresponds to the special $\operatorname{EOS} \nu=1 / 2$, which will be further discussed in Section-6.

\section{Analytic Solutions with Transverse 2D Hubble Embedding and Nontrivial Longitudinal Flow}

In this Section, we try to seek more interesting solutions with nontrivial longitudinal flow, i.e. beyond the simple power law ansatz. Instead of directly going to the second order differential equation in Eq.(26), we return to Eqs.(63) (66) noting the fact that both are homogeneous equations. Motivated by this fact and by the form of 2D and 3D Hubble flows, we start with the following joint ansatz for both the pressure and the velocity field:

$$
\begin{aligned}
& p\left(\tau_{\perp}, z\right)=\tau_{\perp}^{-\frac{a}{1-\nu}} g\left(\frac{z}{\tau_{\perp}}\right) \\
& \bar{v}_{z}\left(\tau_{\perp}, z\right)=f\left(\frac{z}{\tau_{\perp}}\right)
\end{aligned}
$$

The above ansatz particularly features nontrivial dependence on the scaling variable $\xi=\frac{z}{\tau_{\perp}}$ and extra nonscaling time structure in the pressure. It is interesting to note that 2D Hubble flow corresponds to the trivial solution $f=0$ at $a=2$ while the $3 \mathrm{D}$ Hubble flow corresponds to $f=\xi$ at $a=3$, both of which are special cases of the above ansatz.

By inserting the above ansatz to (63) and (66), we obtain two first order ordinary differential equations involving $g(\xi) \equiv g\left(\frac{z}{\tau_{\perp}}\right)$ and $f(\xi) \equiv f\left(\frac{z}{\tau_{\perp}}\right)$. Solving for $f^{\prime}(\xi)$ and $g^{\prime}(\xi)$ from the equations (with' meaning $\frac{d}{d \xi}$ ), we obtain:

\footnotetext{
${ }^{3}$ Of course, the concepts of explosion and implosion depend on the reference point. We will choose the origin as the reference point if not further specified
} 


$$
\begin{aligned}
& f^{\prime}(\xi)=-\frac{\nu\left(1-f(\xi)^{2}\right)\left(2-a+a f(\xi)^{2}-2 \xi f(\xi)\right)}{(\nu-1)(f(\xi)-\xi)^{2}+\nu(\xi f(\xi)-1)^{2}} \\
& g^{\prime}(\xi)=-\frac{g}{1-\nu} \times \\
& \frac{-2 f(\xi)+a f(\xi)+2 \nu f(\xi)-2 a \nu f(\xi)+2 \xi-a \xi-2 \nu \xi+a \nu \xi+a \nu \xi f(\xi)^{2}}{(\nu-1)(f(\xi)-\xi)^{2}+\nu(\xi f(\xi)-1)^{2}}
\end{aligned}
$$

We find that the Eq.(33) involves $f(\xi)$ only and thus decouples from $g(\xi)$. So one may first focus on solving (33) and with $f(\xi)$ obtained one can easily find $g(\xi)$ from integrating Eq. (34). Again one may check that the 2D Hubble flow with $f=0$ at $a=2$ and the 3D Hubble flow with $f=\xi$ at $a=3$ both satisfy the above equations. We observe Eq.(33) and Eq.(34) are invariant under simultaneous transformations: $\xi \rightarrow-\xi, f \rightarrow-f, g \rightarrow g$. This looks like a manifestation of the time inversion symmetry, i.e. $\tau_{\perp} \rightarrow-\tau_{\perp}$, in ideal hydrodynamics, where dissipation terms are absent in stress energy tensor. However, we note $\tau_{\perp}>0$ by definition. The symmetry can be interpretted as parity: $z \rightarrow-z$. It leads to the conclusion that any solution to Eq.(33) and Eq.(34) is accompanied by its parity inversion counterpart. We will identify such pairs in explicit solutions later.

To solve (33), we can also consider it as (inversely) determining $\xi=\xi(f)$ as a function of variable $f$. We can then introduce a new function

$$
t(f) \equiv \frac{f \xi(f)-1}{f-\xi(f)}
$$

and recast (33) into a differential equation for $t(f)$ :

$$
\begin{aligned}
& {\left[\left(1-f^{2}\right)(a f-2 t+a t)\right] \times \frac{d t}{d f}} \\
& \quad-\left[\left(\kappa^{2}-a\right) f+\left(\kappa^{2}+2-a\right) t+(a-1) f t^{2}+(a-3) t^{3}\right]=0
\end{aligned}
$$

In the above we have replaced the E.o.S parameter $\nu$ by $\kappa$ with the relation $\nu=\frac{1}{\kappa^{2}+1}\left(\kappa^{2} \geq 1\right.$ such that $\left.\nu \leq 1 / 2\right)$. In what follows we try to seek solutions $t(f)$ with two different types of ansatz.

\subsection{Solutions from Linear Polynomial Ansatz}

Motivated by the fact that all the coefficients in Eq.(36) are polynomials of both $t$ and $f$, we naturally start with polynomial ansatz for the function $t(f)$. The simplest form is:

$$
t(f)=\alpha f+\beta
$$


Inserting the ansatz into Eq.(36), we obtain

$$
\begin{aligned}
& C_{0}+C_{1} f+C_{2} f^{2}+C_{3} f^{3}=0 \Rightarrow \\
& 0=C_{0}=-\beta\left[2-a+\kappa^{2}+(2-a) \alpha+(a-3) \beta^{2}\right] \\
& 0=C_{1}=a-\kappa^{2}+\left(-2+2 a-\kappa^{2}\right) \alpha+(a-2) \alpha^{2}+(1-a) \beta^{2}+(9-3 a) \alpha \beta^{2} \\
& 0=C_{2}=-\beta \alpha[-4+3 a+(3 a-9) \alpha] \\
& 0=C_{3}=-\alpha(1+\alpha)[a+(a-3) \alpha]
\end{aligned}
$$

The resulting four algebraic equations can be easily solved, with all solutions listed below:

I. $\alpha=0, \beta=0$ leading to

$$
t=0 \rightarrow f=\frac{1}{\xi}, \text { with } a=\kappa^{2}
$$

II. $\alpha=\kappa^{2}, \beta=0$ leading to

$$
t=\frac{a f}{3-a} \rightarrow f=\frac{\left(1+\kappa^{2}\right) \xi \pm \sqrt{\left(1+\kappa^{2}\right)^{2} \xi^{2}-4 \kappa^{2}}}{2 \kappa^{2}}, \text { with } a=\frac{3 \kappa^{2}}{1+\kappa^{2}}
$$

The first solution coincides with the "anti-Hubble" solution found in previous section. The second solution is a new class of solutions. We will return to a detailed discussion of it at the end of this section. There are also nonphysical solutions which we discard, for example the solution $\alpha=-1, \beta=0$ to the set of algebraic equations leads to $t=-f$ and thus $f= \pm 1$ which does not comply with the physical constraint $|f|<1$.

One in principle can use various more general ansatz, for example: (a) polynomials beyond the linear one we used; (b) polynomial types with non-integer powers. These would typically be more complicated but may lead to more solutions. One class we examined is the form $t=\alpha f^{\gamma}+\beta$ with $\gamma$ being any positive real number, which leads to no more solutions other than the ones found above with $\gamma=1$.

\subsection{Solutions from Nonlinear Ansatz}

In this subsection we go beyond the linear polynomial ansatz and show one example of nonlinear ansatz, which lead to interesting new solutions. Motivated by the $1-f^{2}$ term in Eq.(36), we are led to the following nonlinear ansatz:

$$
t(f)=\alpha f+\beta+\frac{\rho f+\lambda}{1-f^{2}}
$$

Furthermore we consider only specific solutions with $a=3$ which has the advantage of "killing" the $t^{3}$ term in the Eq.(36). Inserting this ansatz into the 
equation for $t(f)$ we obtain:

$$
\begin{aligned}
& \frac{1}{1-f^{2}}\left[D_{0}+D_{1} f+D_{2} f^{2}+D_{3} f^{3}+D_{4} f^{4}+D_{5} f^{5}\right]=0 \Rightarrow \\
& 0=D_{0}=(\beta+\lambda)\left(1-\kappa^{2}+\alpha+\rho\right) \\
& 0=D_{1}=3-\kappa^{2}+\left(4-\kappa^{2}\right) \alpha+\alpha^{2}-2 \beta(\beta+\lambda)+\left(4-\kappa^{2}\right) \rho+\rho^{2}+2 \alpha \rho \\
& 0=D_{2}=\beta\left(\kappa^{2}-1-6 \alpha-3 \rho\right)+3 \lambda(2-\alpha) \\
& 0=D_{3}=\kappa^{2}-3+\left(\kappa^{2}-7\right) \alpha-4 \alpha^{2}+2 \beta^{2}+3 \rho-4 \alpha \rho \\
& 0=D_{4}=5 \alpha \beta \\
& 0=D_{5}=3 \alpha(1+\alpha)
\end{aligned}
$$

By solving the above set of algebraic equations we found all the nontrivial and physical solutions listed below:

III. $\alpha=0, \beta=2, \rho=-2, \lambda=-2$ leading to

$$
t=-\frac{2 f}{1-f} \rightarrow f=\frac{1}{2-\xi}, \text { with } \quad a=3 \text { and } \kappa=1
$$

IV. $\alpha=0, \beta=-2, \rho=-2, \lambda=2$ leading to

$$
t=-\frac{2 f}{1+f} \rightarrow f=-\frac{1}{2+\xi}, \text { with } \quad a=3 \text { and } \kappa=1
$$

V. $\alpha=0, \beta=1, \rho=-1, \lambda=-1$ leading to

$$
t=-\frac{f}{1-f} \rightarrow f=\frac{1 \pm \sqrt{5-4 \xi}}{2(\xi-1)}, \text { with } \quad a=3 \text { and } \kappa=2
$$

VI. $\alpha=0, \beta=-1, \rho=-1, \lambda=1$ leading to

$$
t=-\frac{f}{1+f} \rightarrow f=\frac{1 \pm \sqrt{5+4 \xi}}{2(\xi+1)}, \text { with } a=3 \text { and } \kappa=2
$$

VII. $\alpha=0, \beta=0, \rho=-1, \lambda=0$ leading to

$$
t=-\frac{f}{1-f^{2}} \rightarrow f=\left\{\begin{array}{c}
\frac{2}{3 \xi}[1-\zeta(\xi)-\bar{\zeta}(\xi)] \\
\frac{2}{3 \xi}\left[1+\frac{1+i \sqrt{3}}{2} \zeta(\xi)+\frac{1-i \sqrt{3}}{2} \bar{\zeta}(\xi)\right. \\
\frac{2}{3 \xi}\left[1+\frac{1-i \sqrt{3}}{2} \zeta(\xi)+\frac{1+i \sqrt{3}}{2} \bar{\zeta}(\xi)\right.
\end{array}\right]
$$

with $a=3$ and $\kappa=\sqrt{6}$

$$
\text { and } \begin{aligned}
\zeta(\xi) & =\left[-1+\frac{27}{16} \xi^{2}+i \sqrt{1-\left(1-\frac{27}{16} \xi^{2}\right)^{2}}\right]^{1 / 3} \\
\bar{\zeta}(\xi) & =\left[-1+\frac{27}{16} \xi^{2}-i \sqrt{1-\left(1-\frac{27}{16} \xi^{2}\right)^{2}}\right]^{1 / 3}
\end{aligned}
$$


The case for general $a \neq 3$ with the same ansatz (41) is also investigated, in which case a set of 10 algebraic equations result. Many possibilities arise, and we analyzed most of them and found no more solutions, but few of the possibilities lead to really complicated situation that is not analytically tractable. Though an exhaustive investigation of general $a$ is not achieved, it seems most likely there is no solutions other than those listed above with $a=3$. There are certainly many more possible ansatz which could be tested in future works.

\subsection{Discussion of Nontrivial Solutions}

In this section we choose to discuss properties and applicable kinematic domains of solutions II,III,IV,V,VI,VII.

We start with solution-II. It is subject to the physical constraint $|f|<1$ and reality condition of $f$. The applicable kinematic domain of the solution-II given in Eq.(40) is given below (where we already replace $\xi$ by the original coordinate variables $z / \tau_{\perp}$ and also replace $\kappa^{2}$ by $\left.(1-\nu) / \nu\right)$ :

$$
\begin{aligned}
& \bar{v}_{z}=f\left(\frac{z}{\tau_{\perp}}\right) \\
& p=\text { constant } \times \tau_{\perp}^{-3} \frac{f^{\frac{1-3 \nu}{1-\nu}}}{\left(1-f^{2}\right)^{\frac{2-3 \nu}{1-\nu}}} \\
& \text { domain 1: } f=\frac{z / \tau_{\perp}+\sqrt{z^{2} / \tau_{\perp}^{2}-4 \nu(1-\nu)}}{2(1-\nu)} \\
& \text { with } 2 \sqrt{\nu(1-\nu)}<\frac{z}{\tau_{\perp}}<1 \\
& \text { domain 2: } f=\frac{z / \tau_{\perp}+\sqrt{z^{2} / \tau_{\perp}^{2}-4 \nu(1-\nu)}}{2(1-\nu)} \\
& \text { with } \frac{z}{\tau_{\perp}}<-1 \\
& \text { domain 3: } f=\frac{z / \tau_{\perp}-\sqrt{z^{2} / \tau_{\perp}^{2}-4 \nu(1-\nu)}}{2(1-\nu)} \\
& \text { with }-1<\frac{z}{\tau_{\perp}}<-2 \sqrt{\nu(1-\nu)} \\
& \text { domain } 4: f=\frac{z / \tau_{\perp}-\sqrt{z^{2} / \tau_{\perp}^{2}-4 \nu(1-\nu)}}{2(1-\nu)} \\
& \text { with } \frac{z}{\tau_{\perp}}>1
\end{aligned}
$$

In principle, any combination out of the four cases is an allowed solution. Here we will treat them as four independent solutions as there is no overlap in their applicable domains, i.e.they are causally disconnected from each other. Note solutions in domain 1 and domain 3 are related by parity inversion. The same is true for solutions in domain 2 and domain 4 . The transverse part of the flow in all cases is always exploding 2D Hubble by embedding. The longitudinal 
flow in $z$ direction, as indicated by the sign of $f$, is also exploding outward in all four cases, since we have $f>0$ for $\frac{z}{\tau_{\perp}}>0$ and $f<0$ for $\frac{z}{\tau_{\perp}}<0$. The pressure drops as $\tau_{\perp}$ increases keeping $\frac{z}{\tau_{\perp}}$ constant. The solutions in some sense are like shock waves: at each given time moment $\tau_{\perp}$, the flow is in certain domain of the full space; while with changing $\tau_{\perp}$, the spatial shape of the flow profile (in $z$ ) does not change but only has its overall size scale up with $\tau_{\perp}$.

Next we turn to solutions-III and IV. We first note they are related by parity inversion. Therefore, we will concentrate on solution-III only. With physical constraint $|f|<1$ applied, solution-III looks like:

$$
\begin{aligned}
& \bar{v}_{z}=f\left(\frac{z}{\tau_{\perp}}\right) \\
& p=\text { constant } \times \tau_{\perp}^{-6} \frac{1+1 / f}{(1 / f-1)^{3}} \\
& \text { with } \nu=\frac{1}{2} \\
& \text { domain 1: } f=\frac{1}{2-z / \tau_{\perp}} \\
& \text { with } \frac{z}{\tau_{\perp}}>3 \\
& \text { domain } 2: f=\frac{1}{2-z / \tau_{\perp}} \\
& \text { with } \frac{z}{\tau_{\perp}}<1
\end{aligned}
$$

Similar to solution-II, solution-III also contains two causally disconnected pieces. If we shift the scaling variable: $z / \tau_{\perp} \rightarrow z / \tau_{\perp}-2$, which amounts to choosing $z / \tau_{\perp}=2$ as the reference point. The shifted solution $f=-\frac{\tau_{\perp}}{z}$ represents imploding fluid.

The other pair of solutions related by parity inversion is $\mathbf{V}$ and VI. We will focus on solution-V. In terms of original coordinate variables, the solution with physical constraint applied is shown as follows: 


$$
\begin{aligned}
& \bar{v}_{z}=f\left(\frac{z}{\tau_{\perp}}\right) \\
& p=\text { constant } \times \tau_{\perp}^{-15 / 4} \frac{1}{(1 / f-1)^{15 / 8}(1+1 / f)^{5 / 8}} \\
& \text { with } \nu=\frac{1}{5} \\
& \text { domain } 1: f=\frac{1+\sqrt{5-4 z / \tau_{\perp}}}{2\left(z / \tau_{\perp}-1\right)} \\
& \text { with } \frac{z}{\tau_{\perp}}<-1 \\
& \text { domain } 2: f=\frac{1-\sqrt{5-4 z / \tau_{\perp}}}{2\left(z / \tau_{\perp}-1\right)} \\
& \text { with } \frac{z}{\tau_{\perp}}<1
\end{aligned}
$$

The transverse flow is again exploding by embedding, while the longitudinal part differs from solution-II. Note $f$ does not change sign, solution in domain 1 corresponds to fluid moving in the negative $z$ direction, while solution in domain 2 corresponds to fluid moving in the positive $z$ direction. The pressure drops as $\tau_{\perp}$ increases keeping $\frac{z}{\tau_{\perp}}$ constant. They can be interpretted as shock waves with infinite extension.

Finally, we discuss solution-VII. Under the physical constraint $|f|<1$ and reality condition, we are left with:

$$
\begin{aligned}
& \bar{v}_{z}=f\left(\frac{z}{\tau_{\perp}}\right) \\
& p=\text { constant } \times \tau_{\perp}^{-7 / 2}\left(1 / f^{2}-1\right)^{-7 / 4} \\
& \text { with } \nu=\frac{1}{7} \\
& \text { domain } 1: f=f_{1} \equiv \frac{2 \tau_{\perp}}{3 z}\left[1-\zeta\left(z / \tau_{\perp}\right)-\bar{\zeta}\left(z / \tau_{\perp}\right)\right] \\
& \text { with } \frac{z}{\tau_{\perp}}>0 \text { or } \frac{z}{\tau_{\perp}}<0 \\
& \text { domain } 2: f=f_{2} \equiv \frac{2 \tau_{\perp}}{3 z}\left[1+\frac{1+i \sqrt{3}}{2} \zeta\left(z / \tau_{\perp}\right)+\frac{1-i \sqrt{3}}{2} \bar{\zeta}\left(z / \tau_{\perp}\right)\right] \\
& \text { with } 0<\frac{z}{\tau_{\perp}}<1 \text { or }-1<\frac{z}{\tau_{\perp}}<0
\end{aligned}
$$

Let us first comment on the reality of the solution: If $1-\left(1-\frac{27}{16} \xi^{2}\right)^{2}>0$, i.e. the square root in the definition of $\zeta$ and $\bar{\zeta}$ is real, we can specify the argument of the square bracket such that $\zeta$ and $\bar{\zeta}$ are complex conjugate to each other, which guarantees the reality of $f_{1}$ and $f_{2}$. If the square root becomes imaginary, only $f_{1}$ remains real. The parity inversion simply maps $f_{1}$ and $f_{2}$ to themselves. It is 
also worth noting that solution in domain 1 and domain 2 contain discontinuity at $\frac{z}{\tau_{\perp}}=0$. They can be combined to give two smooth solutions at $\frac{z}{\tau_{\perp}}=0$ :

$$
\begin{aligned}
& f= \begin{cases}f_{1} & z / \tau_{\perp}<0 \\
f_{2} & 0<z / \tau_{\perp}<1\end{cases} \\
& f= \begin{cases}f_{2} & -1<z / \tau_{\perp}<0 \\
f_{1} & z / \tau_{\perp}>0\end{cases}
\end{aligned}
$$

This solution is similar to solution-V. It can also be interpretted as shock wave with infinite extension. (60) corresponds to fluid moving in the positive $z$ direction, while (61) corresponds to fluid moving in the negative $z$ direction.

\section{General Scaling Solutions}

In this paper and in previous approaches, quite a few solutions have been found with simple scaling forms for velocity field, it is thus of interest to investigate all possible solutions with the following simple scaling form velocity field (in $t, x, y, z$ coordinates):

$$
v_{x}=\alpha_{x} \frac{x}{t}, v_{y}=\alpha_{y} \frac{y}{t}, v_{z}=\alpha_{z} \frac{z}{t}
$$

The three constants $\alpha_{x, y, z}$ can in principle be different corresponding to anisotropic scaling solutions. These forms include many of the known solutions (cyclic in $x, y, z)$ :

1) $\alpha_{x}=1, \alpha_{y, z}=0-$ Hwa-Bjorken or 1D Hubble flow;

2) $\alpha_{x, y}=1, \alpha_{z}=0-2 \mathrm{D}$ Hubble flow;

3) $\alpha_{x, y, z}=1-3 \mathrm{D}$ Hubble flow;

4) setting $\alpha_{z}=1$ leads to a 1D Hubble embedding in Liao and Koch paper [29], in which a scaling solution $\alpha_{x, y}=-1$ with $\nu=1 / 2$ has been found;

5) setting $\alpha_{x, y}=1$ leads to a 2D Hubble embedding in the present paper, in which another scaling solution $\alpha_{z}=-3$ with $\nu=1 / 2$ has been found.

Beyond the above, it should be mentioned that generalization of spherically symmetric scaling flow in arbitrary $(1+\mathrm{d}) \mathrm{D}$ has been done in [21].

It would be of great interest to exhaust all solutions with the above simple scaling ansatz. To do that, we simply submit the flow field into the hydrodynamics equation in (11) (and using simple flat coordinates $(t, \vec{x})$ ), which leads to 
the following equations for $p(t, x, y, z)$ :

$$
\begin{aligned}
& {\left[h\left(t^{2}-\nu h\right)\right] p_{, t}+\left[h t x \alpha_{x}\right] p_{, x}+\left[h t y \alpha_{y}\right] p_{, y}+\left[h t z \alpha_{z}\right] p_{, z}} \\
& \quad+\left[t\left(\alpha_{s} t^{2}-\zeta_{x} \alpha_{x}^{2} x^{2}-\zeta_{y} \alpha_{y}^{2} y^{2}-\zeta_{z} \alpha_{z}^{2} z^{2}\right)\right] p=0 \\
& \quad\left[h t x \alpha_{x}\right] p_{, t}+\left[h\left(\alpha_{x}^{2} x^{2}+\nu h\right)\right] p_{, x}+\left[h \alpha_{x} \alpha_{y} x y\right] p_{, y}+\left[h \alpha_{x} \alpha_{z} x z\right] p_{, z} \\
& \quad+\left[\alpha_{x} x\left(\kappa_{x} t^{2}-\lambda_{x} \alpha_{x}^{2} x^{2}-\xi_{x}^{-} \alpha_{y}^{2} y^{2}-\xi_{x}^{+} \alpha_{z}^{2} z^{2}\right)\right] p=0 \\
& {\left[h t y \alpha_{y}\right] p_{, t}+\left[h \alpha_{y} \alpha_{x} y x\right] p_{, x}+\left[h\left(\alpha_{y}^{2} y^{2}+\nu h\right)\right] p_{, y}+\left[h \alpha_{y} \alpha_{z} y z\right] p_{, z}} \\
& \quad+\left[\alpha_{y} y\left(\kappa_{y} t^{2}-\xi_{y}^{+} \alpha_{x}^{2} x^{2}-\lambda_{y} \alpha_{y}^{2} y^{2}-\xi_{y}^{-} \alpha_{z}^{2} z^{2}\right)\right] p=0 \\
& \quad\left[h t z \alpha_{z}\right] p_{, t}+\left[h \alpha_{z} \alpha_{x} z x\right] p_{, x}+\left[h \alpha_{z} \alpha_{y} z y\right] p_{, y}+\left[h\left(\alpha_{z}^{2} z^{2}+\nu h\right)\right] p_{, z} \\
& \quad+\left[\alpha_{z} z\left(\kappa_{z} t^{2}-\xi_{z}^{-} \alpha_{x}^{2} x^{2}-\xi_{z}^{+} \alpha_{y}^{2} y^{2}-\lambda_{z} \alpha_{z}^{2} z^{2}\right)\right] p=0
\end{aligned}
$$

In the above, we have introduced a few constants (as certain combinations of $\left.\alpha_{x, y, z}\right)$ :

$$
\begin{aligned}
& \alpha_{s}=\alpha_{x}+\alpha_{y}+\alpha_{z} \\
& \zeta_{x, y, z}=2+\alpha_{s}-2 \alpha_{x, y, z} \\
& \lambda_{x, y, z}=1+\alpha_{s}-\alpha_{x, y, z} \\
& \kappa_{x, y, z}=-1+\alpha_{s}+\alpha_{x, y, z} \\
& \xi_{x, y, z}^{ \pm}=1+2 \alpha_{x, y, z} \pm\left(\alpha_{y, z, x}-\alpha_{z, x, y}\right)
\end{aligned}
$$

and also a coordinate-dependent function

$$
h\left(x^{\mu}\right)=t^{2}-\alpha_{x} x^{2}-\alpha_{y} y^{2}-\alpha_{z} z^{2}
$$

The above derived equations (63)64, 65166) can be recast into the following concise form:

$$
\mathcal{M}^{\mu \nu} \partial_{\nu}(\operatorname{lnp})=B^{\mu}
$$

from which we can explicitly solve out

$$
\partial_{\nu}(\operatorname{lnp})=\mathcal{M}_{\mu \nu}^{-1} B^{\mu}
$$

The full expressions are somewhat long and not included here. In analogy to Section.3, we can apply the compatibility condition, i.e. the commutativity of partial derivatives, which give six constraint equations on $\alpha_{x}, \alpha_{y}$ and $\alpha_{z}$. Among these constraints, only four are independent. In practice, we find it easier to solve the constraint equations by starting from $(\ln p)_{, x, y}=(\ln p)_{, y, x}$ and then deal others subsequently. After tedious calculations, it has been found that an exhaustive list of all possible solutions include the following (all solutions valid subject to cyclic $x \rightarrow y \rightarrow z \rightarrow x$ ):

I. $\alpha_{x}=1, \alpha_{y, z}=0-$ Hwa-Bjorken or 1D Hubble flow;

II. $\alpha_{x, y}=1, \alpha_{z}=0-2 \mathrm{D}$ Hubble flow;

III. $\alpha_{x, y, z}=1-3 \mathrm{D}$ Hubble flow; 
IV. $\alpha_{x}=\alpha_{y}$ and $1+\alpha_{x}+\alpha_{y}+\alpha_{z}=0$ with $\nu=1 / 2$;

V. $\alpha_{x}=0$ and $1+\alpha_{x}+\alpha_{y}+\alpha_{z}=0$ with $\nu=1 / 2$.

The applicable kinematic region for all of the above solutions is simply $\alpha_{x}^{2} x^{2}+$ $\alpha_{y}^{2} y^{2}+\alpha_{z}^{2} z^{2}<t^{2}$, which comes from the constraint that the flow velocity shall be less than the speed of light. We notice that there are no more solution corresponding to general E.o.S parameter $\nu$ except the well-known Hubble flows in various dimensions. While at $\nu=1 / 2$ (which somehow is a saturating extreme), we obtain two classes of solutions, with the solution IV including the solutions found previously as special cases and the solution $\mathbf{V}$ as a completely new class. Note the condition $1+\alpha_{x}+\alpha_{y}+\alpha_{z}=0$ implies the matter is imploding $\left(\alpha_{i}<0\right)$ in at least one of the directions. The Solution $\mathbf{I V}$ and $\mathbf{V}$ represent implosion, but could contain one or two directions that are exploding $\left(\alpha_{i}>0\right)$.

\section{Summary}

In summary, we have investigated various analytic $(1+3) \mathrm{D}$ solutions to relativistic ideal hydrodynamic equations based on known low-dimensional scaling solutions. We first studied solutions with transverse 2D Hubble Embedding, and derived a single ODE Eq.(26) for the remaining longitudinal velocity field. Using this equation we found all solutions with transverse 2D Hubble embedding and power law ansatz (28) for the remaining longitudinal velocity field, as listed in Eqs.(30). To find solutions with transverse 2D Hubble embedding but nontrivial longitudinal flows beyond power law ansatz, is both very interesting and extremely hard, and nevertheless we managed to find a few such solutions corresponding to different physical conditions, as listed in Eqs.(39]40|43/44] 45]46]47). The last question we studied is all possible scaling solutions with the general scaling form in Eq. (62) for the velocity field, to which the complete answer was also found and listed. These analytic solutions describe various examples of explosion, implosion, and shock-wave like processes in relativistic hydrodynamics. Though the initial motivation came from successful numerical hydrodynamic description of heavy ion collisions, the solutions presented in this paper may not directly apply to those but provide useful general indications for similar explosions in heavy ion collisions and also certain astrophysical processes. Admittedly, a lot more work would be needed to reach a better and closer analytic description of the hydrodynamic phenomena relevant for today's relativistic heavy ion collisions.

Acknowledgements: We are grateful to Volker Koch and Edward Shuryak for discussions. The work of S.L. is supported by by the US-DOE grants DEFG02-88ER40388 and DE-FG03- 97ER4014. The work of J.L. is supported by the Director, Office of Energy Research, Office of High Energy and Nuclear Physics, Divisions of Nuclear Physics, of the U.S. Department of Energy under Contract No. DE-AC02-05CH11231. 


\section{References}

[1] L. D. Landau and E. M. Lifshitz, "Fluid Mechanics", 2nd ed., ButterworthHeinemann, 1987.

[2] S. Weinberg, "Gravitation and Cosmology: Principles and Applications of the General Theory of Relativity", John Wiley \& Sons, 1972.

[3] L. D. Landau, Izv. Akad. Nauk SSSR Ser. Fiz. 17, 51(1953). I. M. Khalatnikov, Zhur. Eksp. Teor. Fiz. 27, 529(1954).

[4] E. Shuryak, "The QCD Vacuum, Hadrons and Superdense Matter", 2nd ed., World Scientific Publishing Company, 2004.

[5] E. V. Shuryak, Phys. Lett. B 78, 150 (1978) [Sov. J. Nucl. Phys. 28, 408.1978 YAFIA,28,796 (1978 YAFIA,28,796-808.1978)].

[6] J. Y. Ollitrault, Phys. Rev. D 46, 229 (1992).

[7] D. Teaney, J. Lauret and E. V. Shuryak, Phys. Rev. Lett. 86 (2001) 4783 arXiv:nucl-th/0011058.

[8] P. F. Kolb, P. Huovinen, U. W. Heinz and H. Heiselberg, Phys. Lett. B 500 (2001) 232 arXiv:hep-ph/0012137.

[9] S. A. Voloshin, A. M. Poskanzer and R. Snellings, arXiv:0809.2949 [nucl$\mathrm{ex}]$.

[10] P. F. Kolb and U. W. Heinz, arXiv:nucl-th/0305084. U. W. Heinz, arXiv:0901.4355 [nucl-th]]. D. A. Teaney, arXiv:0905.2433 [nucl-th]]. P. Romatschke, arXiv:0902.3663 [hep-ph]].

[11] T. Schaefer and D. Teaney, arXiv:0904.3107 [hep-ph]].

[12] J. Liao and V. Koch, arXiv:0909.3105 [hep-ph]].

[13] J. Liao and V. Koch, Phys. Rev. Lett. 103 (2009) 042302 arXiv:0902.2377 [nucl-th]].

[14] V. Koch, arXiv:0908.3176 [nucl-th]].

[15] E. V. Shuryak, Prog. Part. Nucl. Phys.53, 273 (2004); Prog. Part. Nucl. Phys. 62, 48 (2009). arXiv:0807.3033 [hep-ph]].

[16] M. Gyulassy and L. McLerran, Nucl. Phys. A 750 (2005) 30 arXiv:nucl-th/0405013. E. V. Shuryak, Nucl. Phys. A 750, 64 (2005) arXiv:hep-ph/0405066. 
[17] J. Liao and E. Shuryak, Phys. Rev. C 75, 054907 (2007); arXiv:hep-ph/0611131. Phys. Rev. Lett. 101, 162302 (2008); arXiv:0804.0255[hep-ph]]. J. Liao and E. V. Shuryak, Phys. Rev. D 73 (2006) 014509 arXiv:hep-ph/0510110. J. Liao and E. V. Shuryak, Nucl. Phys. A 775 (2006) 224 (arXiv:hep-ph/0508035]. J. Liao and E. Shuryak, Phys. Rev. C 77 (2008) 064905 arXiv:0706.4465 [hepph]]. J. Liao and E. Shuryak, Phys. Rev. Lett. 102 (2009) 202302 arXiv:0810.4116 [nucl-th]]. J. Liao and E. Shuryak, arXiv:0804.4890 [hep-ph]]. J. Liao and E. Shuryak, arXiv:0809.2419 [hep-ph]].

[18] M. N. Chernodub and V. I. Zakharov, Phys. Rev. Lett. 98, 082002 (2007). arXiv:hep-ph/0611228.

[19] R. C. Hwa, Phys. Rev. D 10, 2260(1974).

[20] J. D. Bjorken, Phys. Rev. D27, 140(1983).

[21] M. I Nagy, T. Csörgő and M. Csanád, Phys. Rev. C 77, 024908 (2008). arXiv:0709.3677 [nucl-th]].

[22] A. Bialas, R. A. Janik and R. B. Peschanski, Phys. Rev. C 76, 054901 (2007). arXiv:0706.2108 [nucl-th]].

[23] S. Pratt, Phys. Rev. C 75, 024907 (2007) arXiv:nucl-th/0612010.

[24] C. Y. Wong, Phys. Rev. C 78, 054902 (2008); arXiv:0808.1294 [hep-ph]]. arXiv:0809.0517 [nucl-th]].

[25] T. Csörgö, F. Grassi, Y. Hama and T. Kodama, Heavy Ion Phys. A 21, 63 (2004) [Acta Phys. Hung. A 21, 63 (2004)]. [arXiv:hep-ph/0204300].

[26] Yu. M. Sinyukov and I. A. Karpenko, Acta Phys. Hung. A 25, 141 (2006). arXiv:nucl-th/0506002.

[27] T. S. Biró, Phys. Lett. B 474, 21 (2000) arXiv:nucl-th/9911004; Phys. Lett. B 487, 133 (2000) arXiv:nucl-th/0003027.

[28] R. Peschanski and E. N. Saridakis, arXiv:0906.0941 [nucl-th]].

[29] J. Liao and V. Koch, Phys. Rev. C 80, 034904 (2009) arXiv:0905.3406 [nucl-th]]. 